Recepción: 20 / 04 / 2017

Aceptación: 20 / 05 / 2017

Ciencias Jurídicas y Sociales

Publicación: 15 / 06 / 2017

Artículo Científico

\title{
Las obligaciones tributarias y el alto rendimiento deportivo
}

\author{
Tax obligations and high performance sports
}

\section{Passivos e esportes imposto desempenho}

Ángel O. Sisalema-Carrillo ${ }^{\mathrm{I}}$

Borman R. Vargas-Villacres ${ }^{\mathrm{I}}$ I

Correspondencia: editor@polodelconocimiento.com

Abogado de los Juzgados y Tribunales de la Republica del Ecuador; Doctor en Contabilidad y Auditoria; Magister en Tributación y Derecho Empresarial; Universidad Técnica de Ambato.

II. Licenciado en Ciencias de la Educación Profesor de Enseñanza Secundaria en la Especialización de Filosofía y Ciencias Socio Económicas; Abogado de los Juzgados y Tribunales de la Republica del Ecuador; Licenciado en Ciencias Políticas y Sociales; Doctor en Ciencias de la Educación mención Gerencia Educativa; Doctor Dentro del Programa de Formación Inicial y Permanente de Profesionales de la Educación e Innovación Educativa; Doctor en Jurisprudencia; Magister en Desarrollo Educativo; Universidad Técnica de Ambato. 



\section{Resumen}

Los representantes del deporte con excepcionales características de competencia mundial han hecho posible ver sus cualidades en su constante preparación, concentración, desenvolvimiento y participación las competencias, ha hecho posible que sus Clubs deportivos, Federaciones por deporte, Comité Olímpico Ecuatoriano, Ministerios Sectoriales estén pendientes de sus logros los que les atribuye sus merecidos y emotivos reconocimientos económicos, como ingresos extraordinarios para su preparación para próximos eventos, se suman a este logro las marcas publicitarias con incentivos económicos a cambio de mostrar su imagen en los entrenamientos, preparación y competencia.

Esto permite que se genere un ingreso económico adicional o extraordinario para el deportista o deportistas de alto rendimiento por lo que es necesario realizar un análisis económico global y pormenorizado sobre los verdaderos valores que percibe evitando que en lo posterior esta actividad deportiva genere atención o intereses por parte de la administración tributaria por posible elusión o evasión de impuestos.

La evasión fiscal o tributaria considerada como una actividad oculta de los contribuyentes y que la administración tributaria mediante cruce de información o auditorias tributarias, establezcan de estos contribuyentes penalidades llegando incluso a emitir resoluciones preventivas de sanción en procesos de actos administrativos llegando hasta las coactivas. En cambio la elusión considerada como actividad abierta sin control tributario.

El Estado aporta los recursos necesarios planificados para las diferentes disciplinas deportivas reconocidas por el COI, los deportistas de alto rendimiento podrán participar en forma activa y dinámica en las Juegos Olímpicos contempladas en la Ley de Deporte como organismo rector con todas las garantías constitucionales en las participaciones mundiales.

Palabras clave: Evasión, elusión, sujeto pasivo, administración tributaria, alto rendimiento deportivo, impacto económico, sanciones, penalidades, coactiva. 


\section{Abstract}

Representatives of sport with exceptional characteristics of world competition have made it possible to see their qualities in their constant preparation, concentration, development and participation of the competitions, has made it possible for their Sports Clubs, Federations for Sport, Ecuadorean Olympic Committee, Sector Ministries to Their achievements that attribute their deserved and emotional economic recognition, as extraordinary income to prepare for upcoming events, add to this achievement advertising brands with economic incentives in exchange for showing their image in training, preparation and competition.

This allows an additional or extraordinary economic income to be generated for the athlete or highperformance athletes so it is necessary to carry out a comprehensive and detailed economic analysis of the true values he perceives avoiding that in the later this sport activity generates attention or interest for Part of the tax administration for possible circumvention or evasion of taxes.

The tax evasion or tax considered as a hidden activity of taxpayers and that the tax administration through information crossing or tax audits, establish of these taxpayers penalties even going so far as to issue preventive resolutions of sanction in processes of administrative acts reaching to the coercive ones. In contrast the avoidance considered as an open activity without tax control.

The State provides the necessary resources planned for the different sports disciplines recognized by the IOC, high-performance athletes will be able to participate actively and dynamically in the Olympic Games contemplated in the Sports Law as governing body with all the constitutional guarantees in the participations Worldwide.

Key words: Evasion, circumvention, taxpayer, tax administration, high performance sports, economic impact, penalties, penalties, coercive. 


\section{Resumo}

Representantes esporte com características excepcionais de competição global tornaram possível ver as suas qualidades em suas habilidades de preparação, concentração, desenvolvimento e participação constantes, permitiu a sua clubes desportivos, federações desportivas, Comitê Olímpico do Equador, os ministérios sectoriais estão pendentes suas realizações que lhes atribui a sua recompensa financeira merecido e emocional, como receita extraordinária para a preparação para os próximos eventos, adicionar para esta conquista marcas de publicidade com a mudança econômica sua imagem para mostrar no treinamento, preparação e competição incentivos.

Isto permite uma renda adicional ou extraordinário gerado para os atletas atleta ou alto desempenho, por isso é necessário realizar uma análise económica global e detalhada dos verdadeiros valores percebidos prevenção no final deste desporto gera atenção ou interesse parte das autoridades fiscais por possível evasão fiscal ou a evasão.

Fiscal ou evasão fiscal considerada uma atividade oculta do contribuinte ea administração fiscal por verificação cruzada ou auditorias fiscais, estabelecendo penas para esses contribuintes, mesmo para emitir resoluções preventivas de sanção processos administrativos atos coercitivos alcance. Em vez evasão considerada atividade aberta sem controle fiscal.

O Estado fornece os recursos necessários previstos para os vários esportes reconhecidos pelo COI, os atletas de alto rendimento podem participar ativamente e de forma dinâmica nos Jogos Olímpicos contempladas na Lei do Esporte como o órgão com todas as garantias constitucionais nas unidades mundo.

Palavras chave: fraude, evasão, contribuinte, a administração fiscal, desempenho desportivo, impacto econômico, multas, penalidades, coercivas. 


\section{Introducción.}

La presente investigación nace por un interés único y personal, con inclusión profesional por el deporte y con toda la diversificación de conocimiento en el deporte de alto rendimiento, tiene como finalidad dar un aporte para el cumplimiento de obligaciones tributarias de deportistas de alto rendimiento deportivo, así como poder dar un aporte o responder dudas o inquietudes de cómo tributar correctamente analizando los impactos relativos a temas como la elusión y evasión de los impuestos como son: impuesto al valor agregado IVA, Impuesto a la renta anual de personas naturales y a las posibles exenciones de ser el caso.(Definicion Stiglitz, 2000)

“Los impuestos pueden ser de dos clases: Impuestos Directos (sobre las personas físicas y sociedades con personería jurídica) y los Impuestos Indirectos (Bienes y Servicios)”

El deportista profesional actualmente ya no se conforma con éxitos o triunfos morales, sino mas bien con un seguimiento oportuno con preparación a nivel internacional con resultados de calidad y de excelencia, con adecuada capacitación de todas sus responsabilidades y con adecuados presupuestos establecidos para este efecto deportivo por parte de organismos estatales, o con fondos públicos o privados.

Finalmente obtendremos los resultados luego de aplicar las respectivas encuestas a los deportistas clasificados, los mismos que serán tabulados y calculados. 


\section{Materiales y métodos.}

\section{Modalidad Básica de la Investigación}

Como factor predominante, la investigación que se presenta a continuación se realizó bajo enfoques cuantitativo y cualitativo; Podríamos considerar como cuantitativo porque la información obtenida en los CEAR (Centros de Alto Rendimiento) es lugar donde se generan los hechos y al respectivo análisis con cuadros estadístico. Se considera cualitativo en base a los resultados estadísticos que pasan a la criticidad con sustento o soporte para nuestro Marco Teórico, como motor de análisis.

La Administración Tributaria, dentro de sus procesos de verificación, control y determinación de obligaciones tributarias, ha encontrado algunos casos en los cuales hay evidentes y serios indicios del cometimiento de infracciones tributarias; por lo cual es necesario activar mecanismos jurídicos de protección a favor del Estado Ecuatoriano

Bibliográfica - documental.-Se consideran materiales documentales y estadísticas con un trabajo de investigación, los mismos que serán obtenidos de libros referenciales, textos públicos, periódicos, resoluciones jurídicas, así como revistas deportivas, internet y demás documentos legales hasta llegar a la realidad de la investigación, con aplicación interpretativa de la Constitución del Ecuador, Leyes jurídicas, Código Tributario, Código Orgánico Integral Penal, Ley del Deporte, donde rigen las garantías legales de los deportistas desde su iniciación y posterior ingreso al alto rendimiento que se encuentran como tema principal.

La Constitución del Ecuador en su artículo 83 establece como deber y responsabilidad de todo ciudadano: 
“... 15. Cooperar con el Estado y la comunidad en la seguridad social, y pagar los tributos establecidos por la ley...”(SRI)

Aquí se establece el carácter social y solidario de los impuestos

De Campo.-Toda actividad laboral, así como jurídica en este caso que nos ocupa de los deportistas de alto rendimiento y profesionales tiene relación con el Ministerio de trabajo, aplicando el Código del Trabajo con relación laboral de carácter especial.

“A tal efecto, se consideran deportistas profesionales quienes, en virtud de una relación establecida con carácter regular, se dediquen voluntariamente a la práctica del deporte por cuenta y dentro del ámbito de organización y dirección de otro sujeto (un club, una entidad deportiva, una empresa dedicada a la organización de acontecimientos deportivos o cualquier otra empresa) a cambio de una retribución.

Si la relación es esporádica y no regular, queda privada de su carácter especial, lo que no significa que necesariamente quede también privada de su carácter laboral, que se mantendrá si persiste la nota de dependencia”. (http://revistas.um.es/analesderecho/article, 2011)

El campo de la presente investigación tuvo lugar y acceso donde se está generando el problema, para de manera inmediata socializar el tema con los involucrados que pertenecen al llamado Ciclo Olímpico, trasformando la realidad de los deportistas, mediante la exigibilidad de los derechos deportivos.

Como trabajo de investigación de campo se consolidará los valores consignados a cada deportista de forma descriptiva y de las situaciones existentes a este análisis de las situaciones que denotan en cada uno de los beneficiarios con aportes económicos amparados en el Acuerdo 
Ministerial No 1849 donde el Ministerio del Deporte, expide el Reglamento Operativo de ejecución del proyecto al deporte de Alto Rendimiento 2013 - 2016.

\section{Nivel o Tipo de Investigación}

Exploratorio.-De manera exploratoria porque nuestra investigación nos permitirá revisar en forma documentada y sustentadora una introducción visual en las diferentes situaciones que se han venido suscitando en el ámbito de la jurídico -deportivo referente a las asignaciones y aportes económicos para competencias en evento nacionales e internacionales y en diferentes campos donde se practica el deporte de alto nivel de rendimiento y como esto incide estos aportes económicos en el cometimiento de infracciones tributarias (evasión - elusión) cuando estos no han cumplido con los parámetros de declaración de impuestos sean estos, declaración del IVA, Fuente e impuesto a la renta anual.

Descriptiva.-Realizada la investigación de exploración, se iniciará con un análisis de recopilación de la información con la finalidad de socializar y poder establecer procedimientos que se deben mejorar en base a las obligaciones tributarias y poder realizar las declaraciones en forma correcta mensual y anualmente, misma que permitirá hacer comparaciones con deportistas de igual o mejor magnitud que perciben permanentemente aportes económicos con instituciones privadas, organismos públicos y mixtos.

Explicativa.- Producto del análisis se procederá a realizar una nota explicativa después de la investigación donde una vez consolidada la información recopilada con ejemplificación jurídica y se dará a conocer todas las causas y efectos del problema, mismos que ayudarán a aplicar y poder realizar cada declaración como manda la Ley de Régimen Tributario Interno, esto nos ayudará 
entender la problemática por la que atraviesan los contribuyentes por desconocimiento de los ingresos y la obligatoriedad de declarar cumpliendo con la Ley.

Como fundamento de Derecho penal tributario, no es un deber exclusivo del Estado la recaudación de impuestos sino corresponde a todos los contribuyentes y ciudadanos hacerlo en búsqueda de un beneficio común; constituyéndose la Administración Tributaria en instrumento para cumplir con su deber.

\section{Población y Muestra}

Población

Nuestra población deportiva investigada para efectos de muestreo, no es numerosa, y que en base a la creación de una nueva normativa creada en el año 2013 por el Ministerio del Deporte, existen incentivos deportivos niveles de aportes, se procederá a trabajar con todo el universo señalado el mismo que está distribuido en la tabla de incentivos.

Dentro de este estudio se aplicarán a todos los elementos de la población existente, porque su universo es pequeño, evitando realizar algún tipo de muestreo.

\section{LISTADO DE DEPORTISTAS DEL PLAN DE ALTO RENDIMIENTO 2014 - 2015}

\begin{tabular}{|c|c|c|c|c|}
\hline No & DEPORTISTAS & CATEGORIA & DEPORTE & PROVINCIA \\
\hline 1 & CÉSAR DE CESARE & A & CANOTAJE & GUAYAS \\
\hline 2 & ANGGIE AVEGNO & A & CANOTAJE & CARCHI \\
\hline 3 & JORGE BOLAÑOS & A & HOCKEY PATIN & PICHINCHA \\
\hline 4 & INGRID FACTOS & A & HOCKEY PATIN & MANABI \\
\hline 5 & ESTEFANIA GARCIA & A & JUDO & CARCHI \\
\hline 6 & MARLIN VIVEROS & A & JUDO & \\
\hline
\end{tabular}


Las obligaciones tributarias y el alto rendimiento deportivo

\begin{tabular}{|c|c|c|c|c|}
\hline 7 & LENIN PRECIADO & $A$ & JUDO & EL ORO \\
\hline 8 & ANDREA LOOR & A & KARATE & PICHINCHA \\
\hline 9 & FRANKLIN MINA & $A$ & KARATE & PICHINCHA \\
\hline 10 & JAQUELINE FACTOS & $A$ & KARATE & PICHINCHA \\
\hline 11 & DAVID ARROYO & A & LEVANTAMIENTO DE PESAS & ORELLANA \\
\hline 12 & ALEXANDRA ESCOBAR & A & LEVANTAMIENTO DE PESAS & ESMERALDAS \\
\hline 13 & SELEDINA NIEVES & A & LEVANTAMIENTO DE PESAS & ESMERALDAS \\
\hline 14 & SAMANTHA AREVALO & $A$ & NATACION & AZUAY \\
\hline 15 & LISSETH ANTES & $A$ & LUCHA & LOJA \\
\hline 16 & YOAN BLANCO & A & LUCHA & GUAYAS \\
\hline 17 & MARIA SOTOMAYOR & A & RACQUETBALL & AZUAY \\
\hline 18 & MARIA PAZ MUÑOZ & A & RACQUETBALL & AZUAY \\
\hline 19 & FERNANDO RIOS & A & RACQUETBALL & AZUAY \\
\hline 20 & JOSE DANIEL ALVAREZ & A & RACQUETBALL & AZUAY \\
\hline 22 & JONATHAN MARTINETTI & $A$ & VELERISMO & GUAYAS \\
\hline
\end{tabular}

Tabla 1 Listado Deportistas de Alto Rendimiento Deportivo Fuente: Investigador

Muestra

En nuestro universo, no se aplicará fórmula para determinar alguna muestra aleatoria pues nuestra investigación abarcará a toda la población descrita, es decir a todos los deportistas (22 deportistas de alto rendimiento deportivo Clase A) que tienen relación con la aplicación del Modelo de mayor rango económico.

TOTAL DISCIPLINAS

\begin{tabular}{|c|c|}
\hline No & DEPORTE \\
\hline 38 & ATLETISMO \\
\hline
\end{tabular}


Ángel O. Sisalema-Carrillo; Borman R.Vargas-

\begin{tabular}{|c|c|}
\hline 2 & BADMINTON \\
\hline 13 & BOXEO \\
\hline 11 & CANOTAJE \\
\hline 14 & CICLISMO \\
\hline 5 & ECUESTRES \\
\hline 2 & GIMNASIA \\
\hline 10 & HOCKEY PATIN \\
\hline 16 & JUDO \\
\hline 15 & KARATE \\
\hline 21 & LEVANTAMIENTO DE PESAS \\
\hline 9 & NATACION \\
\hline 18 & LUCHA \\
\hline 3 & PENTATLON MODERNO \\
\hline 7 & RACQUETBALL \\
\hline 5 & SQUASH \\
\hline 4 & TAEKWONDO \\
\hline 11 & TENIS DE CAMPO \\
\hline 6 & TENIS DE MESA \\
\hline 1 & TIRO CON ARCO \\
\hline 9 & TIRO DEPORTIVO \\
\hline 6 & TRIATLON \\
\hline 8 & VELERISMO \\
\hline 2 & VOLEIBOL \\
\hline 236 & \\
\hline
\end{tabular}

Tabla 2 Listado de Disciplinas de Alto Rendimiento Fuente: Investigador 


\section{Resultados.}

\section{ANÁLISIS E INTERPRETACION DE DATOS}

El estudio, análisis y colaboración de los deportistas ha permitido obtener los resultados que detallaremos a continuación.

Es necesario indicar que cada una de las preguntas formuladas a los deportistas está relacionada con las variables planteadas en este tema de investigación.

Con el resultado de las encuestas a cada una de las preguntas formuladas, se procedió a la respectiva tabulación, con su respectiva interpretación y análisis e una en una cada pregunta.

PREGUNTA 1. ¿Usted como deportista recibe ingresos extraordinarios a parte de los que le entrega el Ministerio Sectorial?

\begin{tabular}{|c|c|c|}
\hline Alternativa & Frecuencia & Porcentaje \\
\hline SI & 3 & $14 \%$ \\
\hline NO & 19 & $86 \%$ \\
\hline Total & 22 & $100 \%$ \\
\hline
\end{tabular}

Tabla 3 Recibe ingresos Extraordinarios Fuente; lista de verificación

Análisis e Interpretación: Se demuestra que el 14\% de los deportistas encuestados responde que SI que reciben dineros extraordinarios de la empresa privada, que pueden ser como sponsor, gratificaciones o incentivos, mientras que el $86 \%$ responde que NO. 
Estas respuestas individuales a cada uno de los deportistas que se les formula se considera que reciben por participaciones internacionales con aportes de la empresa privada.

PREGUNTA 2. ¿Piensa usted como deportista que debe presentar sus obligaciones tributarias con datos reales de sus ingresos?

\begin{tabular}{|l|l|l|}
\hline Alternativa & Frecuencia & Porcentaje \\
\hline SI & 20 & $91 \%$ \\
\hline NO & 2 & $9 \%$ \\
\hline Total & 22 & $100 \%$ \\
\hline
\end{tabular}

Tabla 4 Obligaciones tributarias con datos reales Fuente; Lista de verificación

Análisis e Interpretación: El 9\% de los deportistas encuestados indican que SI, que si deberían presentar sus obligaciones tributarias como manda la Ley, mientras que el $91 \%$ desconoce del cumplimiento de estas obligaciones.

Es un factor muy delicado de que los deportistas desconocen en alto grado este cumplimiento de obligaciones, ya que su mente y esfuerzo está en sus entrenamientos y participaciones en los diferentes eventos internacionales.

PREGUNTA 3. ¿Cree usted que ofrecer recompensas a quien denuncie el cometimiento de evasión es una solución?

\begin{tabular}{|c|c|c|}
\hline Alternativa & Frecuencia & Porcentaje \\
\hline SI & 13 & $59 \%$ \\
\hline NO & 9 & $41 \%$ \\
\hline Total & 22 & $100 \%$ \\
\hline
\end{tabular}

Tabla 5 Denuncia de evasión es una solución

Fuente; Lista de verificación 
Análisis e Interpretación: El 59\%de los deportistas encuestados nos indican que SI están de acuerdo en ofrecer recompensa de los evasores tributarios, mientras que el $41 \%$ de los encuestados NO están de acuerdo en este procedimiento.

Es necesario indicar que la Administración tributaria ofrece incentivos a quienes denuncien sobre el cometimiento de evasión en el cumplimiento de estas obligaciones tributarias.

PREGUNTA 4. ¿Como deportista de alto rendimiento conoce usted en qué consisten los términos de elusión o evasión tributaria?

\begin{tabular}{|c|c|c|}
\hline Alternativa & Frecuencia & Porcentaje \\
\hline SI & 15 & $68 \%$ \\
\hline NO & 7 & $32 \%$ \\
\hline Total & 22 & $100 \%$ \\
\hline
\end{tabular}

\section{Tabla 6 Conoce términos de Evasión y Elusión. \\ Fuente; Lista de verificación}

Análisis e Interpretación: El 68\% de los deportistas encuestados nos proporcionan el resultado de que SI conoce el termino de evasión, mientras que el 32\% dicen NO conocer los términos descritos.

Como medida de conocimiento los deportistas encuestados conocen el término evasión, por lo que estamos en buen camino sobre el tema a investigar.

PREGUNTA 5. ¿Cree usted que la evasión o elusión es un problema de cultura y necesitan capacitación?

\begin{tabular}{|c|c|c|}
\hline Alternativa & Frecuencia & Porcentaje \\
\hline SI & 21 & $71 \%$ \\
\hline NO & 1 & $29 \%$ \\
\hline
\end{tabular}


Ángel O. Sisalema-Carrillo; Borman R.Vargas-

\section{\begin{tabular}{|l|l|l|} 
Total & 22 & $100 \%$ \\
\hline
\end{tabular}}

Tabla 7 La Evasión y Elusión es problema de cultura.

Fuente; Lista de verificación

Análisis e Interpretación: El 64\% del personal encuestado indica que SI conoce que la institución cuenta con políticas y procedimientos, mientras que el 36\% desconoce de la existencia de las mismas.

Se puede considerar que los empleados y funcionarios o responsables directos del otorgamiento de créditos saben a ciencia cierta sobre la importancia de contar con políticas y procedimientos para el mejoramiento del otorgamiento de créditos.

PREGUNTA 6. ¿Cree usted que se debería calificar como evasora las personas que dan realizando las declaraciones?

\begin{tabular}{|c|c|c|}
\hline Alternativa & Frecuencia & Porcentaje \\
\hline SI & 6 & $27 \%$ \\
\hline NO & 16 & $73 \%$ \\
\hline Total & 22 & $100 \%$ \\
\hline
\end{tabular}

\section{Tabla 8 Son evasores las personas que dan declarando} Fuente; Lista de verificación

Análisis e Interpretación: El 27\% de los deportistas encuestados indican que SI se deberían considerar como evasores a las personas que realizan o dan realizando las obligaciones tributarias, mientras que el $73 \%$ indican que NO se deberían considerar como evasores.

Es necesario indicar que algunos deportistas no poseen RUC, lo que le impide realizar con el cumplimiento tributario.

PREGUNTA 7. ¿Como deportista cree que cumplir con las obligaciones tributarias con datos irreales es algo involuntario? 


\begin{tabular}{|c|c|c|}
\hline Alternativa & Frecuencia & Porcentaje \\
\hline SI & 4 & $18 \%$ \\
\hline NO & 18 & $82 \%$ \\
\hline Total & 22 & $100 \%$ \\
\hline
\end{tabular}

Tabla 9 Obligaciones tributarias con datos reales. Fuente; Lista de verificación

Análisis e Interpretación: El 18\% de los deportistas encuestados indican que SI, que es algo involuntario ya que existen documentos que no poseen ya que la mayoría de ellos son extranjeros, mientras que el $82 \%$ no lo consideran.

En esta pregunta, los deportistas indican que los documentos en su mayoría son por compras en el extranjero y que es necesario que se ilustre de cómo se puede considerar estos documentos en las obligaciones tributarias.

PREGUNTA 8. ¿Usted como deportista cree que las empresas auspiciantes deberían firmar contrato con los deportistas y no con las federaciones por deporte?

\begin{tabular}{|c|c|c|}
\hline Alternativa & Frecuencia & Porcentaje \\
\hline SI & 9 & $41 \%$ \\
\hline NO & 13 & $59 \%$ \\
\hline Total & 22 & $100 \%$ \\
\hline
\end{tabular}

Tabla 10 Firma de contratos con deportistas. Fuente; Lista de verificación

Análisis e Interpretación: El 41\% de los deportistas encuestados indican que SI que se deberían firmar los contratos cuando se tratan de auspicios o sponsor porque es necesario que el deportista sea el beneficiario, mientras que el 59\% está en desacuerdo de la firma de los contratos.

Se puede considerar que los deportistas de alto rendimiento mientras más competencias a nivel internacional participen, son mucho mejor porque ellos deberían ser los beneficiarios de estos valores económicos. 
PREGUNTA 9. ¿Conoce usted como deportista las nuevas sanciones estipuladas en el COIP por defraudación tributaria?

\begin{tabular}{|c|c|c|}
\hline Alternativa & Frecuencia & Porcentaje \\
\hline SI & 2 & $9 \%$ \\
\hline NO & 20 & $91 \%$ \\
\hline Total & 22 & $100 \%$ \\
\hline
\end{tabular}

\section{Tabla 11 Conoce la defraudación tributaria dictada por el COIP Fuente; Lista de verificación}

Análisis e Interpretación: El 9\% de los deportistas encuestados indican que SI que conocen algo de las nuevas leyes penales y que eso les inquieta en algún momento les vaya a molestar, mientras que el $91 \%$ desconoce de la existencia de las nuevas leyes tributarias y penales.

Las reformas que constantemente se realizan a las leyes y códigos es para el cumplimiento de todos y mejorar los procedimientos que realizan diferentes actividades para el bienestar de todos.

PREGUNTA 10. ¿Como deportista cree que mientras más participaciones internacionales le representan más dinero con auspicio de la empresa pública y privada?

\begin{tabular}{|c|c|c|}
\hline Alternativa & Frecuencia & Porcentaje \\
\hline SI & 3 & $14 \%$ \\
\hline NO & 19 & $86 \%$ \\
\hline Total & 22 & $100 \%$ \\
\hline
\end{tabular}

Tabla 12 Participaciones internacional hay mas auspicios Fuente; Lista de verificación

Análisis e Interpretación: El 14\% de los deportistas encuestados indican que SI que por sus participaciones en eventos internacionales con destacadas actuaciones reciben muy buenos incentivos y gratificaciones económicas, mientras que el 86\% NO reciben algún tipo de incentivo. 
Las participaciones de los deportistas en eventos internacionales organizados por el COI permiten que los deportistas mientras más destacados resultados tengan, mejores incentivos les espera por parte de la empresa privada como parte de Sponsor publicitarios.

PREGUNTA 11. ¿Cree usted que el declarar sus obligaciones tributarias, el Fisco distribuye equitativamente en el deporte?

\begin{tabular}{|c|c|c|}
\hline Alternativa & Frecuencia & Porcentaje \\
\hline SI & 15 & $68 \%$ \\
\hline NO & 7 & $32 \%$ \\
\hline Total & 22 & $100 \%$ \\
\hline
\end{tabular}

Tabla 13 Distribución equitativa por parte del fisco

Fuente; Lista de verificación

Análisis e Interpretación: El 68\% de los deportistas encuestados indica que SI se distribuye equitativamente los ingresos del estado en el deporte, mientras que el $32 \%$ NO cree que lo distribuye.

Es necesario mencionar que el Estado en el presupuesto general, distribuye equitativamente los recursos dotando de centros de Alto rendimiento deportivo en 5 CEARS del país muy bien equipados para la práctica y entrenamiento deportivo.

PREGUNTA 12. ¿Cree usted que el deporte que practica le está generando buenos ingresos económicos de la empresa pública y privada? 
Ángel O. Sisalema-Carrillo; Borman R.Vargas-

\begin{tabular}{|c|c|c|}
\hline Alternativa & Frecuencia & Porcentaje \\
\hline SI & 9 & $41 \%$ \\
\hline NO & 13 & $59 \%$ \\
\hline Total & 22 & $100 \%$ \\
\hline
\end{tabular}

Tabla 14 Su deporte le genera buenos ingresos

Fuente; Lista de verificación

Análisis e Interpretación: El 41\% de los deportistas de Alto rendimiento encuestados mencionan que SI reciben incentivos por parte de la empresa privada, mientras que el 59\% mencionan que NO reciben.

Se puede considerar que los deportistas de alto rendimiento deportivo se sientes motivados en sus participaciones ya que reciben estímulos por parte de la empresa privada.

\section{VERIFICACIÓN DE HIPÓTESIS}

La verificación de la hipótesis se sigue pasos recomendados por estadísticos:

1. Establecer la hipótesis nula y alternativa.

La hipótesis planteada para la investigación es:

Obligaciones Tributarias y el Alto Rendimiento Deportivo

* Variable Independiente: Obligaciones Tributarias

* Variable Dependiente: Deporte de Alto Rendimiento

Planteamiento de la:

Hipótesis nula H0 e Hipótesis alternativa H1.

H0: Las Obligaciones Tributarias NO inciden en el Alto Rendimiento Deportivo 
H1: Las Obligaciones Tributarias inciden en el Alto Rendimiento Deportivo

De esta manera se establece la hipótesis matemática:

$$
\begin{aligned}
& H_{0}: f_{o}=f_{e} \\
& H_{1}: f_{o} \neq f_{e}
\end{aligned}
$$

2. Establecer el nivel de significancia

Para la presente investigación se utiliza el 0.025 de nivel de significancia, que corresponde al $99.75 \%(0,975)$ de nivel de confianza de la información.

3. Seleccionar el estadístico de prueba

En la recolección de información se clasificó dos preguntas de índole cuantitativa, se considera una comprobación para métrica que permita medir los resultados obtenidos, esperando comprobar las diferencias entre la teoría y los hallazgos obtenidos.

Se utilizará el chi-cuadrado que nos permite comprobar las dos variables y analizar su relación.

$$
\chi^{2}=\sum \frac{\left(f_{o}-f_{e}\right)^{2}}{f_{e}}
$$

La comprobación se realizara aplicando el estadígrafo a las siguientes preguntas:

\section{PREGUNTA 9. ¿Conoce usted como deportista las nuevas sanciones estipuladas en el COIP} por defraudación tributaria? 
Ángel O. Sisalema-Carrillo; Borman R.Vargas-

\begin{tabular}{|c|c|c|}
\hline Alternativa & Frecuencia & Porcentaje \\
\hline SI & 2 & $9 \%$ \\
\hline NO & 20 & $91 \%$ \\
\hline Total & 22 & $100 \%$ \\
\hline
\end{tabular}

Tabla 15 Conoce la defraudación tributaria dictada por el COIP.

Fuente; Lista de verificación

PREGUNTA 12. ¿Cree usted que el deporte que practica le está generando buenos ingresos económicos de la empresa privada?

\begin{tabular}{|c|c|c|}
\hline Alternativa & Frecuencia & Porcentaje \\
\hline SI & 9 & $38 \%$ \\
\hline NO & 13 & $62 \%$ \\
\hline Total & 22 & $100 \%$ \\
\hline
\end{tabular}

Tabla 16 Conoce la defraudación tributaria dictada por el COIP.

Fuente; Lista de verificación

Con estas DOS preguntas se construye una tabla de frecuencias que relacione la hipótesis con las 2 variables:

Se procede a continuación a realizar la comprobación de la hipótesis mediante la aplicación del Chi Cuadrado.

NIVEL DE SIGNIFICACION Y GRADOS DE LIBERTAD.

Distribución Muestral

$$
\mathrm{gl}=(\mathrm{k}-1) *(\mathrm{j}-1) \quad \mathrm{gl}=(2-1) *(2-1)
$$

Donde: $\quad \mathrm{gl}=(1) *(1)$

$\mathrm{gl}=$ Grado de libertad $\quad \mathrm{gl}=1$ 
$\mathrm{j}=$ Filas de la tabla

$\mathrm{k}=$ Columnas de la tabla

Nivel de Significación $\quad$ X20.025 $=5,024$

Se utilizará el nivel $\infty=0.025$

Descripción de la Población.

La muestra que es de 22deportistas de Alto Rendimiento Deportivo, es decir 22 respuestas a la propuesta planteada.

Especificación del Estadístico.

De acuerdo a la tabla de contingencia $2 \times 2$ utilizaremos la formula.

$\mathrm{X} 2=\sum(\mathrm{O}-\mathrm{E}) 2$

$\mathrm{X} 2=$ Chi $\mathrm{o}$ Ji cuadrado

$\sum=$ Sumatoria.

$\mathrm{O}=$ Frecuencia Observadas.

$\mathrm{E}=$ Frecuencias Esperadas

OBSERVADO
\begin{tabular}{|l|c|c|c|}
\hline PREGUNTA 9 & 2 & NO & TOTAL \\
\hline PREGUNTA 12 & 9 & 20 & 22 \\
\hline \multirow{2}{*}{ TOTAL } & 11 & 33 & 22 \\
\cline { 2 - 4 } & 0,25 & 0,75 & 44 \\
\hline
\end{tabular}


Ángel O. Sisalema-Carrillo; Borman R.Vargas-

ESPERADO
\begin{tabular}{|l|c|c|c|}
\hline PREGUNTA 9 & 5,5 & 16,5 & 22 \\
\hline PREGUNTA 12 & 5,5 & 16,5 & 22 \\
\hline TOTAL & 11 & 33 & 44 \\
\hline
\end{tabular}

Tabla 17 Cálculo Matemático

Fuente: Análisis Estadístico

\begin{tabular}{|c|c|c|c|}
\hline PREGUNTA 9 & 2,23 & 0,74 & \\
\hline PREGUNTA 12 & 2,23 & 0,74 & \\
\hline TOTAL & 4,45 & 1,48 & 5,94 \\
\hline
\end{tabular}

Tabla 18 Cálculo del Chi Cuadrado

Fuente: Análisis Estadístico

REGLA DE DECISIÓN

Si X2 $<5,024$, aceptar H0, caso contrario rechazar

Entonces 5,940 > 5,024; por tanto rechazar H0 y aceptar H1

Definición de la zona de rechazo

Zona de Aceptación H0

Zona de Rechazo H1 


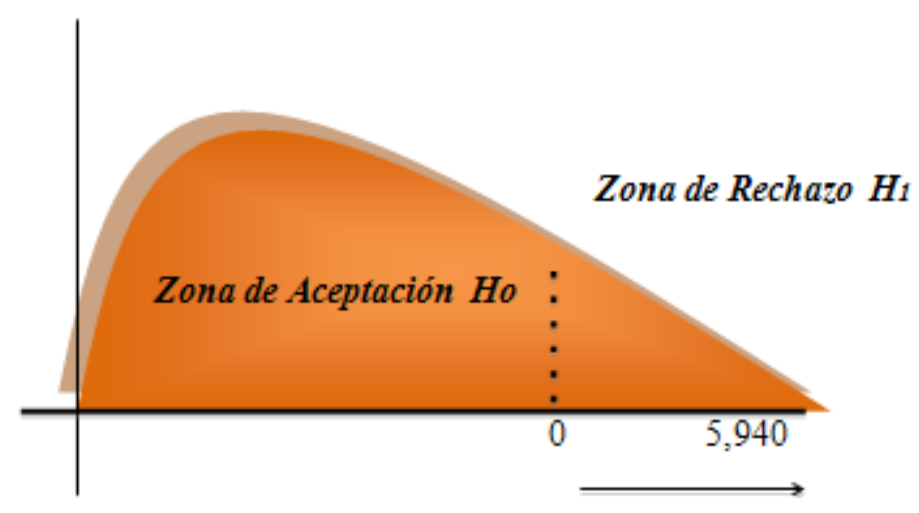

Gráfico 1 Definición zona de rechazo. Fuente: Lista de verificación

De lo cual:

Mediante el cálculo realizado, a nivel de significación del 0,025\%, se obtuvo un valor de Xc $=5.940$ y un valor de $\mathrm{Xt}=5.024$; por lo que se rechaza la Hipótesis Nula (H0) que dice "Las Obligaciones Tributarias NO inciden el Alto Rendimiento Deportivo; por consiguiente se acepta la Hipótesis Alternativa (H1) "Las Obligaciones Tributarias inciden en el Alto Rendimiento Deportivo". 


\section{Conclusiones.}

Realizadas todas las investigaciones referentes al problema planteado, se obtienen las siguientes conclusiones:

- Existe gran porcentaje de desconocimiento por parte de los deportistas de Alto Rendimiento sobre el cumplimiento de sus obligaciones tributarias y de la Ley en particular.

- $\quad$ No existe por parte del Ministerio Sectorial o Clubes privados, ilustración o capacitación profesional ni planificada y que además sea permanente sobre estas obligaciones tributarias que se aplican a los ingresos, egresos mensuales y anuales que han generado como ingreso fijo o variable que sirven de base para el cumplimiento tributario.

- $\quad$ Los deportistas de Alto Rendimiento tanto los avalizados por el Ministerio del Deporte como del deporte profesionales saben que deben cumplir con este requisito como contribuyentes, pero su desconocimiento afectaría gravemente su participación en el caso de que la Administración tributaria crece información financiera de sus ingresos. 


\section{Bibliografía.}

Medina, Remigio; "Guía para retener el impuesto a la renta e IVA”, editorial pedagógica Freire, 2008.

Corporación de Estudios y Publicaciones, "Código Tributario", Impresión Talleres de la Corporación de Estudios y Publicaciones, Edición 2014.

Jiménez, C.; Logroño, M. y Otros, “Módulo de Tutoría 1”, editorial, año 2001.

Ley del Deporte, ediciones jurídicas 2010.

Carta Olímpica, “Comité Olímpico Internacional”, Suiza 2004,

Benegas Lynch, A y Diana, R (2000). "Sistemas Tributarios. Un Análisis Torno al Caso Argentino" Revista libertas 33. Instituto Universitario ESEADE.

Cowell, F.A. (1990) “Tax Sheltering and the cost of evasión”. Oxford Economic Paper, vol.42, pp,231-243.

Esteller - More , A.(2004). "Tax Evasión in Interrelated Taxes" University deBarcelona and Institud d"Ecomimia de Barcelona" (IEB), Document de treball No 2004/2.

Vallejo Ricaurte, I (2014). ¿Quién soy yo, andinista ecuatoriano.

www.ivanvallejo.com

Pedrosa Sáenz, R. "El Impacto del Deporte en la Economía: Problemas de medición” Universidad de Valladolid, Revista Asturiana de Economía RAE No 26-2003.

Auditores y Asesores Gerenciales, "Defraudación Tributaria" (2014).

www.smsecuador.ec

Robalino,L - Observatorio Iberoamericano de Protección de Datos, "El Observatorio" 01/02/2015.Oiprodat.com "Reserva y Sigilo" - "la idea información Legal”

Velásquez. (C) (2015). Universidad Católica de Guayaquil, Revista Jurídica

Atocha Morales, V. (2010), "EL delito Tributario en la legislación Ecuatoriana” Salesiana Ecuador.

Cáceres Contreras, F. (2012), "Incongruencias en el principio de irretroactividad en el código tributario", Universidad José Antonio Páez, I.F. Asesoría y Servicios C.A. C.I.V-5.667.503.

Servicio de Rentas Internas, Fraude Tributario (2015), Fundamentos del Derecho Penal.www.sri.gob.ec Acero, R. M. (2008). Área de Alto Rendimiento Deportivo. Murcia, España: Cultura, Ciencia y Deporte. Bergman, M., \& Nevarez, A. (2005). ¿EVADIR O PAGAR IMPUESTOS? UNA APROCIMACION A LOS MECNISMOS SOCIALES DE CUMPLIMIENTO. Mexico: Política y Gobierno.

CARACUEL, J., \& BOHÓRQUEZ, R. (2012). Rendimiento deportivo, estilos de liderazgo y evitación experiencial en jóvenes futbolistas almerienses. Palma de Mallorca, España: Revista de Psicología del Deporte.

Castilla, F. J., \& León, P. d. (2008). FISCALIZACION TRIBUTARIA. Santa Cruz, Bolivia: Revista Boliviana de Derecho.

Cruz, J. Á. (2009). Fraude fiscal y lavado de capitales. Santiago: Política criminal. 
JARAMILLO, C. y. (2008). El impacto del Impuesto al Valor Agregado sobre el gasto en Colombia. Colombia: Lecturas de Economía.

Luna, G. A. (2014). Ecuador: economía y política de la revolución ciudadana, evaluación preliminar. Boyacá, Colombia: Revista Apuntes del CENES.

Montilla, J. A. (2014). El entrenamiento deportivo en el siglo XXI. Murcia: Cuadernos de Psicología del Deporte. 